\title{
Seismic analysis of plane linear systems with semi-rigid connections
}

\author{
S. Zdravkovic, D. Zlatkov, B. Mladenovic \& M. Mijalkovic \\ Civil Engineering and Architectural Faculty of Nis, Serbia
}

\begin{abstract}
In this paper forming of the stiffness and mass matrices for a bar with semi-rigid connections depending on the level of rigidity of the joint connection will be presented. These two matrices are introduced into well-known computer programs to be modified for the dynamic design of a plane linear system whose connections are semi-rigid. The influence of semi-rigid connections on the change of dynamic characteristics is illustrated using numerical examples. Horizontal seismic forces and maximal horizontal displacements of a frame are computed according to Serbian regulations, based on the circle frequencies and vibration periods of free horizontal frame vibrations calculated for different levels of connection rigidity. The results obtained are presented in diagrams. This paper will also briefly present the results of an integral experimental investigation of seismic performances of the prefabricated reinforced concrete structural system AMONT, developed in Serbia and intended for construction of various types of modern industrial halls. The study has been carried out for the purpose of attesting the structural behaviour in seismic regions, particularly its specific connections and links. It is concluded from the results obtained that the rigidity level of connections should not be neglected, particularly in the analysis of prefabricated structures. The design allowing for semi-rigid connections is especially significant in earthquake engineering, because seismic forces cause the joint connections to become slack, which has not been adequately taken into consideration in up-to-date dynamic analysis.
\end{abstract}

Keywords: semi-rigid connections, stiffness matrix, consistent mass matrix, circle frequency, vibration period, pre-cast reinforced concrete structural system, seismic design. 


\section{Introduction}

The first step in structural analysis is the choice of the so-called idealized design model, which is used for the approximation of the considered structure. Structure geometry, the manner of support, connections of members in joints and load, as well as mechanical properties of the structure materials, are idealized by the model. It is always required that an idealized model as far as possible approximates the real structure and that it is simple and convenient for the practical design of the structure. In the classical theory of structures, connections of members in joints of linear systems are the most often idealized as ideally pinned or absolutely rigid.

Many worldwide researches, based on numerical simulations and experimental results, having been carried out during the last 20 years, indicated that a great number of connections of members in joints of linear systems cannot be classified either as ideally pinned or as absolutely rigid. It is noticed that the level of rigidity of connection is of particular significance in the case of pre-cast structures, because even a low level of rigidity of pre-cast connections affects the redistribution of action effects, the critical load value and the buckling length of the members, as well as the basic dynamic properties of the structure.

The design procedure for structures with semi-rigid connections under dynamic (particularly seismic) load, which is the subject of this paper, is based on the classical deformation method. Having in mind that matrix formulation of a problem is convenient for structural analysis, it is applied to the design of the considered systems.

\section{Interpolation functions for member with semi-rigid connections}

In the case of a straight member bending in plane, the relationship between displacement $\boldsymbol{v}(\boldsymbol{x})$ of whichever point of a member axis and parameter of displacements at the member ends can be the most easily obtained starting from homogeneous differential equation of bending:

$$
\mathrm{EI} \frac{d^{4} v(x)}{d x^{4}}=0
$$

whose solution can be written as a polynomial of the third order, which follows:

$$
v(x)=\alpha_{1}+\alpha_{2} x+\alpha_{3} x^{2}+\alpha_{4} x^{3} .
$$

Coefficients $\alpha_{i}(i=1,2,3,4)$ are defined from boundary conditions at the member ends. Interpolation functions with a shape of Hermit's polynomials determined for a fixed-end member are given in (Sekulovic [1]). In the case of a member with semi-rigid connections at the ends $i$ and $k$, interpolation functions (2) can be derived from differential equation (1) and boundary conditions. 
When unit translation $\mathrm{q}_{1}=1$ is applied to the joint $i$ of a member, while all other generalized displacements are equal to zero, what follows can be written according to the fig. 1.:

$$
\alpha_{i k}^{*}=\left[\mu_{i k}-\left(1-\mu_{i k}\right) \mu_{k i} \frac{b_{i k}}{a_{i k}}\right] \frac{1}{l} ; \quad \alpha_{k i}^{*}=\left[\mu_{k i}-\left(1-\mu_{k i}\right) \mu_{i k} \frac{b_{i k}}{a_{k i}}\right] \frac{1}{l}
$$

where $\mu_{\mathrm{ik}}$ and $\mu_{\mathrm{ki}}$ are rigidity level of joint connections at the ends of a member, which can be determined numerically or experimentally.

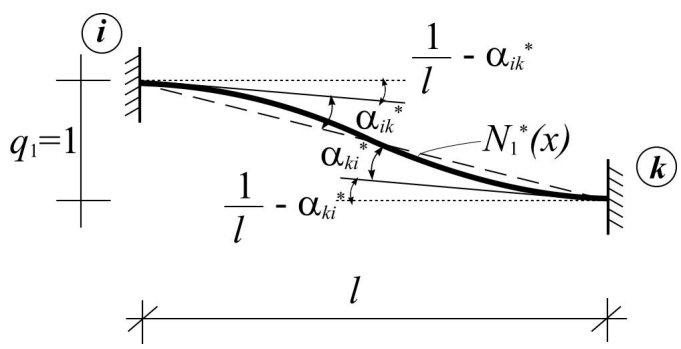

Figure 1: $\quad$ The state $\mathrm{q}_{1}=1.0$.

Boundary conditions for semi-rigid connections at the ends $i$ and $k$ of a member $i k$, from which coefficients $\alpha_{\mathrm{i}}(\mathrm{i}=1,2,3,4)$ appearing in expression (2) can be determined, are written in the following form:

$$
x=0\left\{\begin{array}{c}
v(x)=v_{i}=\alpha_{1}=1 \\
\varphi(x)=\varphi_{i}=\alpha_{2}=-\left(\frac{1}{l}-\alpha_{i k}^{*}\right)
\end{array} \quad x=l\left\{\begin{array}{c}
v(x)=v_{k}=1+\alpha_{2} l+\alpha_{3} l^{2}+\alpha_{4} l^{3}=0 \\
\varphi(x)=\varphi_{k}=\alpha_{2}+2 \alpha_{3} l+3 \alpha_{4} l^{2}=-\left(\frac{1}{l}-\alpha_{k i}^{*}\right)
\end{array}\right.\right.
$$

Then, according to (2), the first element of the matrix of interpolation functions is:

$$
N_{1}^{*}(x)=v(x)_{q_{1}=1}=1-\left(\frac{1}{l}-\alpha_{i k}^{*}\right) x-\frac{2 \alpha_{i k}^{*}+\alpha_{k i}^{*}}{l} x^{2}+\frac{\alpha_{i k}^{*}+\alpha_{k i}^{*}}{l^{2}} x^{3} .
$$

Interpolation function $N_{1}^{*}$ represents Hermit's polynomial of the first order and its diagram is shown in fig. 1 . In the limit case when a member is rigidly connected at its ends $i$ and $k$ (rigidly fixed-end member), that is $\mu_{i k}=\mu_{k i}=1$, expression (5) has already known value (Sekulovic [1]).

Similarly, in the case when unit rotation $\mathrm{q}_{2}=1$ of the joint $i$, unit translation $\mathrm{q}_{3}=1$ and unit rotation $\mathrm{q}_{4}=1$ of the joint $k$ are applied separately, while all of the others generalized displacements are equal to zero, interpolation functions $\boldsymbol{N}_{2}^{*}$, $\boldsymbol{N}_{3}^{*}$ and $\boldsymbol{N}_{4}^{*}$, respectively, can be determined, so that the matrix of interpolation functions can be shown in the following form:

$$
N^{*}=\left[\begin{array}{llll}
N_{1}^{*}(x) & N_{2}^{*}(x) & N_{3}^{*}(x) & N^{*}{ }_{4}(x)
\end{array}\right],
$$

where 


$$
\begin{aligned}
& N_{2}^{*}(x)=\mu_{i k} x-\frac{2 \mu_{i k}-\mu_{k i}+\alpha_{k i}^{*} l}{l} x^{2}+\frac{\mu_{i k}-\mu_{k i}+\alpha_{k i}^{*} l}{l^{2}} x^{3}, \\
& N_{3}^{*}(x)=\left(\frac{1}{l}-\alpha_{i k}^{*}\right) x+\frac{2 \alpha_{i k}^{*}+\alpha_{k i}^{*}}{l} x^{2}-\frac{\alpha_{i k}^{*}+\alpha_{k i}^{*}}{l^{2}} x^{3}, \\
& N_{4}^{*}(x)=\left(\mu_{i k}-\alpha_{i k}^{*} l\right) x-\frac{2 \mu_{i k}+\mu_{k i}-2 \alpha_{k i}^{*} l}{l} x^{2}+\frac{\mu_{i k}+\mu_{k i}-\alpha_{k i}^{*} l}{l^{2}} x^{3} .
\end{aligned}
$$

Matrix $N^{*}$ is the matrix of interpolation functions or the matrix of shape functions for semi-rigidly fixed-end member. Interpolation function $N^{*}{ }_{\mathrm{m}}(x)$ represents an elastic line of a semi-rigidly fixed-end member due to generalized displacement $\mathrm{q}_{\mathrm{m}}=1(\mathrm{~m}=1,2,3,4)$ while all of the others generalized displacements are $\mathrm{q}_{\mathrm{n}}=0, \mathrm{n} \neq \mathrm{m}$.

\section{Stiffness matrix of semi-rigidly fixed-end member}

The stiffness matrix of a semi-rigidly fixed-end member is obtained after the second derivatives of interpolation functions have been determined and looks like:

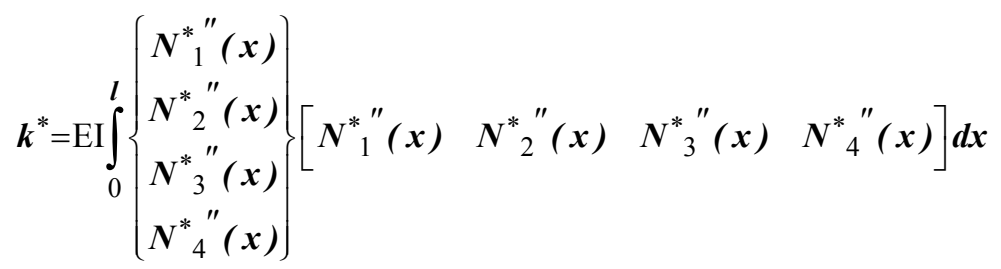

When the axial forces effect on deformation is taken into account, the stiffness matrix of a semi-rigidly fixed-end member can be written as follows:

$$
\boldsymbol{k}^{*}=\left[\begin{array}{cccccc}
\frac{\mathrm{EF}}{l} & 0 & 0 & \frac{\mathrm{EF}}{l} & 0 & 0 \\
& \boldsymbol{k}_{11}^{*} & \boldsymbol{k}_{12}^{*} & 0 & \boldsymbol{k}_{13}^{*} & \boldsymbol{k}_{14}^{*} \\
& & \boldsymbol{k}_{22}^{*} & 0 & \boldsymbol{k}_{23}^{*} & \boldsymbol{k}_{24}^{*} \\
& & & \frac{\mathrm{EF}}{l} & 0 & 0 \\
& & & & \boldsymbol{k}_{33}^{*} & \boldsymbol{k}_{34}^{*} \\
\operatorname{sim} . & & & & & \boldsymbol{k}_{44}^{*}
\end{array}\right],
$$

where 


$$
\begin{aligned}
& \boldsymbol{k}_{11}^{*}=\frac{4 \mathrm{EI}}{l}\left[\alpha_{i k}^{* 2}+\alpha_{i k}^{*} \alpha_{k i}^{*}+\alpha_{k i}^{* 2}\right], \\
& \boldsymbol{k}_{12}^{*}=\frac{2 \mathrm{EI}}{l}\left[2\left(\alpha_{i k}^{*} \mu_{i k}+\alpha_{k i}^{* 2} l-\alpha_{k i}^{*} \mu_{k i}\right)-\alpha_{i k}^{*} \mu_{k i}+\alpha_{i k}^{*} \alpha_{k i}^{*} l+\alpha_{k i}^{*} \mu_{i k}\right], \\
& \boldsymbol{k}_{13}^{*}=-\frac{4 \mathrm{EI}}{l}\left[\alpha_{i k}^{* 2}+\alpha_{i k}^{*} \alpha_{k i}^{*}+\alpha_{k i}^{* 2}\right]=-k_{11}^{*}, \\
& \boldsymbol{k}_{14}^{*}=\frac{2 \mathrm{EI}}{l}\left[2\left(\alpha_{i k}^{*} \mu_{i k}-\alpha_{k i}^{* 2} l+\alpha_{k i}^{*} \mu_{k i}\right)+\alpha_{i k}^{*} \mu_{k i}-\alpha_{i k}^{*} \alpha_{k i}^{*} l+\alpha_{k i}^{*} \mu_{i k}\right] .
\end{aligned}
$$

The expressions for $\boldsymbol{k}_{22}^{*}, \boldsymbol{k}_{23}^{*}, \boldsymbol{k}_{24}^{*}, \boldsymbol{k}_{33}^{*}, \boldsymbol{k}_{34}^{*}, \boldsymbol{k}_{44}^{*}$ are derived as functions of $\alpha_{i k}^{*}, \alpha_{k i}^{*}, \mu_{i k}, \mu_{k i}$ too, Zlatkov [2].

\section{Consistent mass matrix of a semi-rigidly fixed-end member}

In deriving the motion equations of a member, the mass matrix can be adopted as consistent mass matrix or as concentrated masses matrix. Consistent mass matrix is a symmetrical and positively definite square matrix of order $n$, where $n$ is the number of degrees of freedom. The form of the mass matrix $\boldsymbol{m}$ is the same as that one of the stiffness matrix $\boldsymbol{k}$.

Starting from interpolation functions for a semi-rigidly fixed-end member, given in (5) and (7) the elements of a consistent mass matrix can be derived in the following form:

$$
\begin{gathered}
\boldsymbol{m}_{\boldsymbol{m} \boldsymbol{n}}^{*}=\rho \mathrm{F} l \int_{0}^{l} N_{\boldsymbol{m}}^{*}(x) N_{n}^{*}(x) d x, \quad \boldsymbol{m}, \boldsymbol{n}=1, \ldots, 4 \\
\boldsymbol{m}^{*}=\left[\begin{array}{llll}
\boldsymbol{m}^{*} & \boldsymbol{m}_{12}^{*} & \boldsymbol{m}_{13}^{*} & \boldsymbol{m}^{*}{ }_{14} \\
\boldsymbol{m}_{21}^{*} & \boldsymbol{m}^{*}{ }_{22}^{*} & \boldsymbol{m}_{23}^{*} & \boldsymbol{m}^{*}{ }_{24} \\
\boldsymbol{m}_{31}^{*} & \boldsymbol{m}_{32}^{*} & \boldsymbol{m}_{33}^{*} & \boldsymbol{m}_{34}^{*} \\
\boldsymbol{m}_{41}^{*} & \boldsymbol{m}_{42}^{*} & \boldsymbol{m}_{43}^{*} & \boldsymbol{m}_{44}^{*}
\end{array}\right] .
\end{gathered}
$$

Introducing (5) and (7) into (10), after multiplication and integration, the following terms are obtained:

$$
\begin{aligned}
& m_{11}^{*}=\frac{\rho \mathrm{F} l}{420}\left[140+\left(42 \alpha_{i k}^{*}-28 \alpha_{k i}^{*}\right) l+\left(4 \alpha_{i k}^{* 2}+4 \alpha_{k i}^{* 2}-6 \alpha_{i k}^{*} \alpha_{k i}^{*}\right) l^{2}\right], \\
& \boldsymbol{m}_{22}^{*}=\frac{\rho \mathrm{F} l}{420}\left[4\left(\mu_{\boldsymbol{i} \boldsymbol{k}}^{2}+\mu_{\boldsymbol{k} i}^{2}+\alpha_{\boldsymbol{k} \boldsymbol{i}}^{* 2} l^{2}\right)+6\left(\mu_{\boldsymbol{i} \boldsymbol{k}} \mu_{\boldsymbol{k} \boldsymbol{i}}-\mu_{\boldsymbol{i} \boldsymbol{k}} \alpha_{\boldsymbol{k} \boldsymbol{i}}^{*} l\right)-8 \mu_{\boldsymbol{k} \boldsymbol{i}} \alpha_{\boldsymbol{k} \boldsymbol{i}}^{*} l\right] l^{2}, \\
& \boldsymbol{m}_{33}^{*}=\frac{\rho \mathrm{F} l}{420}\left[140-\left(28 \alpha_{i k}^{*}-42 \alpha_{k i}^{*}\right) l+\left(4 \alpha_{i k}^{* 2}+4 \alpha_{k i}^{* 2}-6 \alpha_{i k}^{*} \alpha_{k i}^{*}\right) l^{2}\right] \text {, } \\
& \boldsymbol{m}_{44}^{*}=\frac{\rho \mathrm{F} l}{420}\left[4\left(\mu_{i k}^{2}+\mu_{\boldsymbol{k} i}^{2}+\alpha_{\boldsymbol{k} i}^{* 2} l^{2}\right)+6\left(-\mu_{i k} \mu_{\boldsymbol{k} i}+\mu_{\boldsymbol{k} i} \alpha_{i k}^{*} l\right)-8 \mu_{i k} \alpha_{i k}^{*} l\right] l^{2},
\end{aligned}
$$

as well as $\boldsymbol{m}_{12}^{*}, \boldsymbol{m}_{13}^{*}, \boldsymbol{m}_{14}^{*}, \boldsymbol{m}_{23}^{*}, \boldsymbol{m}_{24}^{*}, \boldsymbol{m}_{34}^{*}$ (Zlatkov [2]). 


\section{Some results of the experimental investigation of precast reinforced concrete structure connections}

For the purpose of attesting of a prefabricated structure, it is enormously important to know the behaviour of its joints and links in linear and deeply nonlinear domain. Taking into account uniqueness and specificity of applied connections, these data could be obtained only by quasi-static nonlinear tests up to the failure by use of the full-scale connection models. The subject of our investigation is the pre-cast reinforced concrete (RC) structural system AMONTKrusce, developed in Serbia, which contains many different connections that could be treated. In this phase of investigation laboratory tests of the AMONT characteristic joints and links under simulated adequate load: connection of the corner column to the beam of the floor structure, connection of the floor element with the beam of the floor structure, as well as connection column to foundation were carried out. The bearing capacity of the weaker place of the corner column, where it is connected to the beam of the floor structure, and bearing capacity of the beam strengthened with connection to the floor element were tested too (Ristic et al. [3]).

Worthwhile experimental results were obtained from these tests, which enabled successful analytical verification of the system stability and publishing of the corresponding attested documentation.

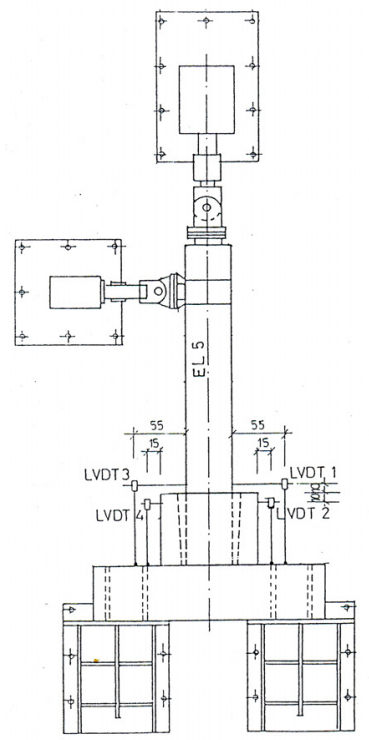

Figure 2: Disposition of the experimental model of the connection column to the foundation with the equipment used.

The results of only one test of the connection column to foundation are presented. Bearing capacity and deformability of this connection of pre-cast RC column $50 / 50 \mathrm{~cm}$ to foundation glass was investigated. Static vertical axial force 
and transverse increasing cyclic force up to the failure were applied as loading. The input axial force simulates an expected gravity load in the considered cross section of the column during exploitation. Disposition of the model with equipment used for the quasi-static test is shown in fig. 2.
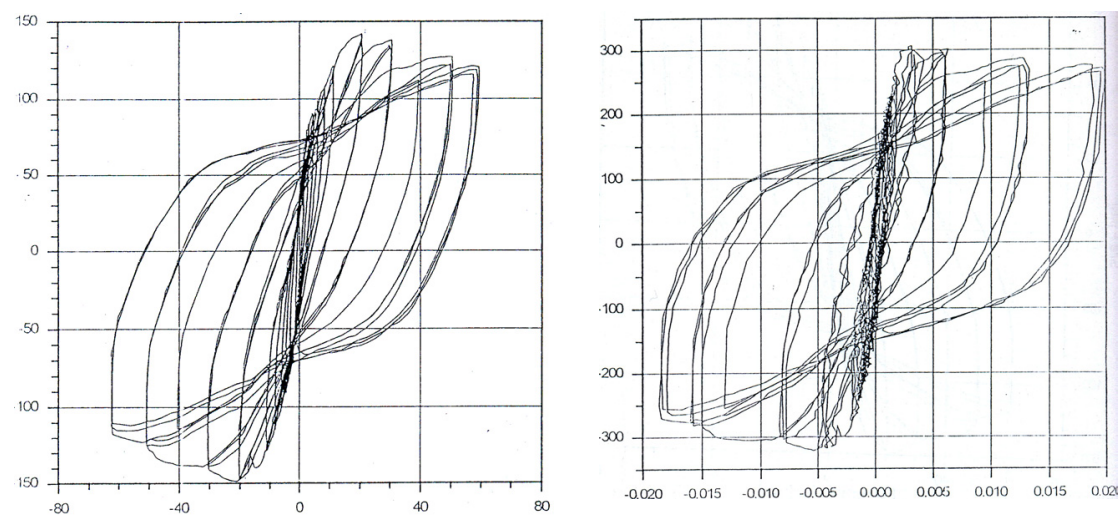

Figure 3: Relation cyclic force-displacement of the column top and relation moment-curve (base LVDT-1 and LVDT-3).

The two-sign moment at the connection column to the foundation glass is simulated by applying cyclic displacement at the column end. The amplitude of the column top displacement was increased successively monotonously up to nonlinear domain of behaviour of the treated connection and the column as well.

Relations force-displacement and moment-curve at the connection column to foundation are shown in fig. 3 .

\section{An example of seismic design of a system with semi-rigid connections}

The well-known computer program STRESS is intended for linear elastic analysis of plane or space structures. There are some programs, which are developed based on STRESS as independent for the purpose of further processing. At the Civil Engineering Faculty in Nis, Serbia, Prof. M. Stankovic has evaluated the program SASS, which is intended for seismic analysis of structures according to Serbian code and enables the calculation of basic dynamical characteristics and seismic action and effects (Stankovic et al. [4]).

Design of structures with semi-rigid connections differs from the standard procedure only in commands containing data about members, i.e. it is necessary to form stiffness matrices of members. Instead of giving cross-section characteristics of prismatic members (area and moment of inertia for the main axis) through the command MEMBER PROPERTIES PRISMATIC, properties 


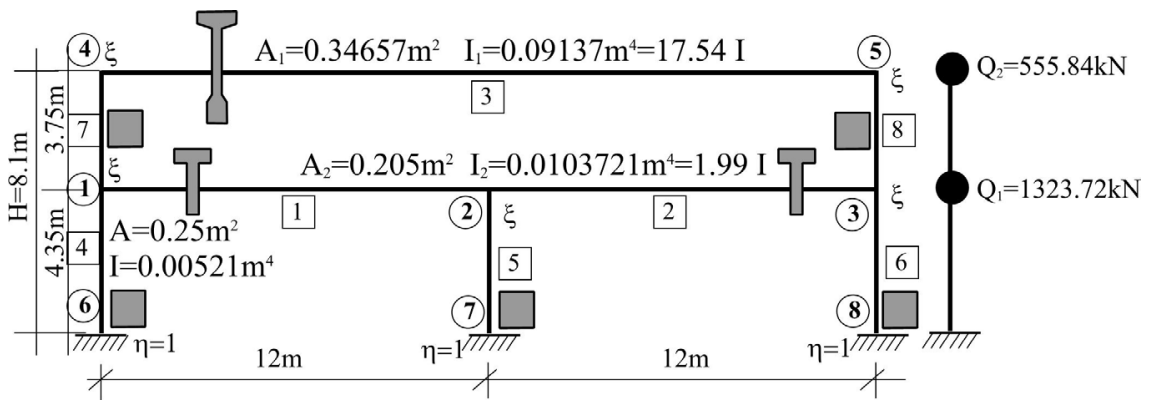

Figure 4: $\quad$ Static scheme and dynamical model of a RC frame of the AMONT prefabricated structural system.

of a member are described through the command STIFFNESS GIVEN, in which case the basic stiffness matrix elements for semi-rigidly connected member are input directly.

As an illustration of this application to frames with semi-rigid connections, the frame structure, presented in fig. 4 is considered. It is a two-floor RC frame of the AMONT prefabricated structural system, Morava Krusce, Serbia, with a span of $24 \mathrm{~m}$, the column cross section $50 \times 50 \mathrm{~cm}$ and the beam cross sections as shown at fig. 4. Based on the results of previously mentioned tests of the connections, it is evident that the connection column to foundation is almost absolutely rigid and because of that the rigidity level is adopted as $\mu_{61}=\mu_{72}=\mu_{83}=\eta=1$ (fixed-end member), while the connection beam to column behaves as $75 \%$ fixed, so it is adopted $\mu_{12}=\mu_{21}=\mu_{23}=\mu_{32}=\mu_{45}=\mu_{54}=\mu_{41}=\mu_{53}=\mu_{27}=\xi=0.75$. For the purpose of comparing dynamic characteristics, as well as displacement of the structure top, depending on the rigidity level of the connections beam to column, the calculation is carried out for three examples of frames. It is taken $\eta=1$ for column to foundation connection in all cases, and in each example different value for $\xi$, i.e. $0,0.75,1$.

According to the expressions (9), for geometrical characteristics of the members of the system shown in fig. 4, the elements of base stiffness matrices are calculated. For example, stiffness sub-matrix (Stankovic et al. [4]) for members 1 and 2 is:

$$
\boldsymbol{k}_{1}^{*}=\boldsymbol{k}_{2}^{*}=\left[\begin{array}{ccc}
580833.3 & 0 & 0 \\
0 & 1606.5 & -9639.4 \\
0 & -9639.4 & 82547.2
\end{array}\right] \text {. }
$$

This numerical data is used for defining the member properties in input file (SASS) as follows:

1 STIFFNESS GIVEN 580833.3 0. 0. 0. 1606.5 -9639.4 0. -9639.4 82547.2

For other members the same calculation has been carried out (Zlatkov [2]).

The part of results of the seismic design according to Serbian Regulation is shown in diagrams below: 

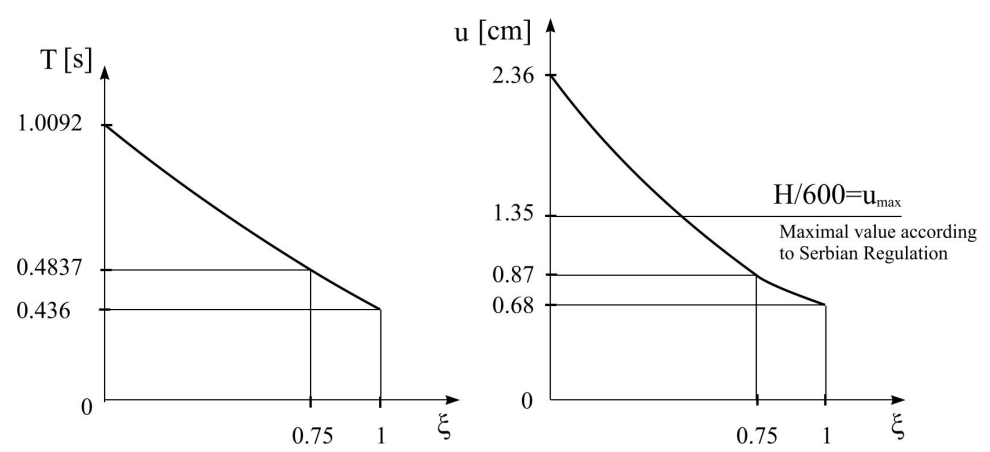

Figure 5: $\quad$ Frame vibration period $\mathbf{T}$ and horizontal displacement $\mathbf{u}$ of the frame top for different rigidity level connections.

\section{Conclusion}

It can be concluded from the results obtained that the structure with an absolutely rigid beam to column connections is too rigid, while that one with pinned connections is too flexible and its period of vibration and displacements exceeded the values allowed by the Serbian Regulations.

Design of seismic resistant structures has to be a compromise between rigidity and flexibility; that means the structure has to be moderately flexible. The example with semi-rigid beam to column connections, where the adopted level of rigidity of connection is $\xi=0,75$, fulfil this requirement because its period of vibration and displacement at the top of the structure are in the frame of values allowed by the Serbian Regulation. This fact is very important in the case of pre-cast reinforced concrete structures, particularly because it is simpler to construct a semi-rigid connection than a rigid one.

Design allowing for semi-rigid connections is especially significant in earthquake engineering because seismic forces cause the joint connections to become slack. The rigidity level of connections should not be neglected, in seismic design, particularly in the case of pre-cast reinforced concrete structures.

\section{Acknowledgements}

This research is supported by the Ministry of Science of the Republic of Serbia, within the framework of the project Experimental and theoretical research of real connections at reinforced concrete and composite structures under static and dynamic loading, No 16001, for the period 2008-2010.

\section{References}

[1] Sekulovic, M., Matrix analysis of structure, Gradjevinska knjiga, Beograd,

[2] Zlatkov, D., Analiza linijskih sistema sa polukrutim vezama stapova $u$ cvorovima, 1-233, Nis, 1998. 
[3] Ristic, D., Micov, V., Zisi, N., Dimitrovski, T., Attesting of Static and Dynamic Stability of the Typified Modulus of a Hall Program of the Precast RC Structural System AMONT-Krusce IZIIS Skopje, 1998.

[4] Stankovic, S., Djordjevic, Dj., Stress, Programski sistem za staticki proracun inzenjerskih konstrukcija-uputstvo za koriscenje programa, Trion Computers, Nis, 1991.

[5] Milicevic, M., Zdravkovic, S., Zlatkov, D., Matrix analysis of systems with semi-rigid connections of members, Proceeding of $21^{\text {st }}$ Yugoslav congress of theoretical and applied mechanics, pp. 265-268, Nis, 1995.

[6] Milicevic, M., Zdravkovic, S., Folic, R., Zlatkov, D., Significance and part of elastic connections of members with joints in earthquake engineering, $11 W C E E, 6$, pp. 23-28, Acapulco, Mexico, 1996.

[7] Milicevic, M., Zdravkovic, S., Folic, R., Zlatkov, D., Theoretical basis and dynamic design of the systems with semi-rigid connections of members with joints, $11 W C E E$, 6, pp. 23-28, Acapulco, Mexico, 1996.

[8] Milicevic, M., Zdravkovic, S., Zlatkov, D., Kostadinov, B., Matrix Formulation of Design and Testing of Structures with Semi-Rigid Connections, Structural Engineers World Congress, pp. 266, San Francisco, California, 1998.

[9] Milicevic, M., Zdravkovic, S., Zlatkov, D., Kostadinov, B., Seismic analysis of structures with semi-rigid connections, 11. EECEE, Paris, 1998.

[10] Milicevic, M., Zdravkovic, S., Zlatkov, D., Kostadinov, B., Dynamic Analysis and Testing of Structures with Semi-rigid Connections, Workshop Computational Structural Dynamics, IZIIS Skopje, Makedonija, 2001.

[11] Zdravkovic, S., Zlatkov, D., Kostadinov, B., Experimental static and dynamic investigation of industrial halls and their connections in the full scale, chapter in Monograph: Theoretical and experimental investigations of elasto-plastical behaviour of structures, pp. 167-178, Civil engineering and architectural faculty of Nis, Serbia, Nis, 2006. 